\title{
La promoción y la enseñanza de las habilidades del pensamiento profundo y visible en las sesiones de Educación Física en Educación Primaria The promotion and teaching of deep and visible thinking skills in Physical Education sessions in Primary Education
}

\author{
Manuel Cañas Encinas, Ruth Pinedo González, Noelia García-Martín \\ Universidad deValladolid (España)
}

\begin{abstract}
Resumen. El siglo XXI demandadesarrollar en el alumnado competenciasvinculadas con el pensamiento crítico, la resolución de problemas, la creatividad, etc. Por ello, enseñar a pensar de forma eficaz debe ser el eje vertebrador de la acción educativa para conseguir que el alumnado desar rolle aprendizajes profundos. Diversas herramientas metodológicas se pueden emplear para la consecución de este fin, entre ellas el enfoque del pensamiento visible. El objetivo de este estudio es conocer la percepción del profesorado sobre la promoción y la enseñanza del pensamiento en Educación Física (EF, en adelante) de Educación Primaria. Se haseguido un diseño cualitativo fenomenológico mediantelarealización de entrevistas semiestructuradas a profesores de EF vinculados con la etapa de Educación Primaria. Los principales resultados reflejan que el profesorado considera necesario potenciar el pensamiento del al umnado en $\mathrm{EF}$, aunque a veces no se le otorga demasiada importancia debido a la herencia negativa del área, a lafalta de formación docente y a la inseguridad del profesorado. Asimismo, predomina el desconocimiento del pensamiento visible, el cual puede convertirse en un marco global desde el cual instaurar una cultura del pensamiento en las sesiones deEF, alavez quese enseñaal alumnado a pensar de formaeficaz. Por ello, sefinal izael artículo real izando diversas recomendaciones para introducir el enfoque señalado en las sesiones de EF.
\end{abstract}

Palabras clave: pensar, Educación Física, Educación Primaria, pensamiento visible.

\begin{abstract}
A competence-based education model for 21st century requires to develop critical thinking, problem solving and creativity. A principal objective of a quality curriculum is teaching to think effectively to develop deep learnings. Several methodologies and strategies can promote deeper learnings in schools, as the visible thinking approach. In light of this, the present study aims to explore teachers' perceptions of the promotion and teaching of thinking in Physical Education (PE) in Primary Education. The research involved a qualitative phenomenological design that employed semi-structured interviews with a purposeful sample of experienced teachers linked to Primary Education level. O verall, teachers consider it necessary to strengthen the thinking of the students in EF, although sometimes it is not given much importance due to the negative legacy of the area, thelack of teacher training and the insecurity of the teaching staff. The majority of teachers reported that they were unaware of the visible thinking approach. This approach could become a global framework to promote a culture of thinking in EF. Therefore, the article finishes with recommendations to introduce the visible thinking approach into EF sessions.
\end{abstract} Keywords: thinking, physical education, primary education, visible thinking

\section{Introducción}

La sociedad del conocimiento del siglo XXI demanda fomentar en el alumnado competencias vinculadas al desarrollo del pensamiento y dela metacognición (D ede, 2010; Perkins, 2016; Scott, 2015; Voogt \& Pareja, 2012). Ejemplo de ello es el proyecto Assessment $\&$ Teaching of 21st Century Skills (ATC21s, 2014), donde se identifican diversas maneras de pensar, tales como: la creatividad y la innovación, la resolución de problemas, el pensamiento crítico y aprender a aprender. Todo esto cobra especial relevancia debido a que el aprendizaje es

Fecha recepción: 09-10-20. Fecha de aceptación: 08-01-21

$M$ anuel Cañas Encinas

manubernardos97@gmail.com el resultado del pensamiento (Perkins, 2016).

León-Díaz, Arija-M ediavilla, Fernando-Martínez \& Santos-Pastor (2020) defienden el uso de enfoques educativos participativos para fomentar una actitud activa, reflexiva y comprensiva por parte del alumnado en EF, lo que favorece que tengan éxito en las clases de EF (Cabañete, Tesouro, Puigggali \& Zagalaz, 2020). En este sentido, Ritchhart, Church \& Morrison (2014) exponen que la palabra «pensar» es una de las más frecuentes en el aula por parte de los docentes. Sin embargo, estos autores se preguntan cómo esta palabra es interpretada por el alumnado y si les conduce a alguna acción, pues «losdocentes piden asus estudiantesque piensen todo el tiempo, pero nunca han dado un paso atrás para considerar qué es lo que quieren específicamente que los alumnos hagan mentalmente cuando se les de- 
manda pensar» (Ritchhart et al., 2014, p. 38). ¿Q ué piensa el alumnado en EF? ¿Cómo promover y capturar pensamientos de orden superior en el alumnado y transferir estos procesos cognitivos a diversos contenidos? (Hamada, 2015).

Por tanto, el primer paso para conseguir que el pensamiento del alumnado sea eficaz es enseñarles a pensar (Liu, M cBride, Ziang \& Scarmardo, 2018). Johnson (2003) identifica tres perspectivas desde las cuales enseñar a pensar: la separación del pensamiento, es decir, la enseñanza de las habilidades del pensamiento al margen de los contenidos curriculares; la inmersión del pensamiento, basada en introducir al al umnado a un clima que invite a la reflexión desde los contenidos curriculares; y la infusión del pensamiento, desde la cual se fusiona la enseñanza explícita de las habilidades del pensamiento con los contenidos de lasáreas curriculares. El hecho de enfrentar al alumnado a situaciones que promuevan el pensamiento no garantiza el desarrollo de un pensamiento eficaz. Como afirman Swartz, Costa, Beyer, Reagan \& Kallick (2017), «la enseñanza del pensamiento dentro de cada asignatura aumenta la eficiencia a la hora de pensar y también hace que se retengan mejor los contenidos estudiados» (p. 42), ya que dichas habilidades deben adquirirse en un contexto de aprendizaje que permitasu uso (Báez \& O nrubia, 2016). Por ello, la perspectiva más adecuada es la infusión del pensamiento (Swart et al., 2017).

En este sentido, Pill \& SueSee (2017) y Hamada (2015) recogen varias herramientas para fomentar el pensamiento crítico en EF, siendo una de ellas el enfoque del pensamiento visible, el cual encaja en la perspectivabasada en lainfusión del pensamiento (Ritchhart, 2015; Ritchhart et al., 2014; Salmon, 2015; Swart et al., 2017).

Este enfoque fue iniciado por investigadores del Proyecto Zero delaU niversidad de H arvard (Salmon, 2015). Hace referencia a «cualquier tipo de representación observable que documente y apoye el desarrollo de las ideas, preguntas, razones y reflexiones en desarrollo de un individuo o grupo» (Tishman \& Palmer, 2005, p. 2). Este proyecto se compone de diferentes ramas centra das en la mejora de los procesos de enseñanza-aprendizaje, entre ellas se encuentra la línea de investigación que persigue que el alumnado aprenda a pensar de forma eficaz (Perkins, 2016). Para ello, se defiende la necesidad de hacer visible el pensamiento, puesto que generalmente es un proceso que ocurre de forma invisible, es decir, no se verbaliza o expresa (Ritchhart \& Perkins, 2008).
Desde el pensamiento visible se defiende que los docentes deben realizar hincapié en la promoción y en la enseñanza de las habilidades o movimientos del pensamiento durantesussesiones con el alumnado (Ritchhart et al. , 2014). Algunos de estos procesos cognitivos son: (a) observar de cerca y describir qué hay ahí, (b) construir explicaciones e interpretaciones, (c) razonar con evidencias, (d) establecer conexiones, (e) tener en cuenta diferentes puntos de vista y perspectivas, (f) captar lo esencial y llegar a conclusiones, (g) preguntarse y hacer preguntas y (h) descubrir la complejidad e ir más allá de la superficie. Estos movimientos son fundamentales paralacomprensión, la resolución de problemas, latoma de decisiones y emitir juicios (Pinedo, Cañas, García y García, 2019; Ritchhart, 2015).

Generar una cultura del pensamiento implica facilitar un espacio donde el pensamiento es valorado, visibilizado y promovido en el quehacer diario del aula de forma individual y de manera colectiva (Salmon, 2008). Para ello, se deben contemplar ocho fuerzas culturales (Ritchhart, 2015): las expectativas, el lenguaje, el tiempo, el modelado, las oportunidades, las interacciones, el ambiente y las rutinas del pensamiento.

Con respecto a la última fuerza cultural señalada, las rutinas del pensamiento se conciben desde una triple perspectiva (Ritchhart et al., 2014): como herramientas, por el hecho de apoyar el desarrollo de los diferentes movimientos del pensamiento por parte del alumnado durante las sesiones; como estructuras, pues los diferentes pasos de cada rutina actúan como un anda miaje natural que conduce al alumnado hacia niveles más complejos de pensamiento; y como patrones de comportamiento, como consecuencia de que su utiliza ción regular permite que sean interiorizadas como modos de actuación ante los contenidos de aprendizaje o su transferencia a situaciones fuera del aula. Estas rutinas pueden perseguir diferentes objetivos didácticos, como presentar y explorar ideas, sintetizar y organizar ideas, o profundizar en ellas (Ritchhart et al., 2014).

Las rutinas del pensamiento se apoyan en organiza dores gráficos para evitar que los pensamientos de una persona puedan perderse, caer en el olvido o no seguir una estructura lógica (M orales \& Restrepo, 2015). Además de estructurar la reflexión, estos organizadores visibilizan el pensamiento a través de la documentación, es decir, el registro de las ideas, de las discusiones, de las reflexiones o de las preguntas que surjan durante el proceso de aprendizaje (Salmon, 2008). Esta documentación puede realizarse a través de audios, vídeos, 
dibujos o de forma escrita. Pérez-Pueyo \& Hortigüela (2020) se preguntan cómo se evidencia el aprendizaje del alumnado en EF. Estos autores plantean la utiliza ción de herramientas que permitan a los docentes constatar el nivel de logro de los contenidos trabajados. El uso de rutinas del pensamiento puede convertirse en una herramienta para conseguir el objetivo menciona do (Ritchhart, 2015).

Las contribuciones de las rutinas del pensamiento son diversas: (a) facilitan la comunicación, el aprendiza je y el pensamiento; (b) aumentan el repertorio de estrategias cognitivas; (c) apoyan la autodirección del alumnado durante su aprendizaje; y (d) fomentan las disposicioneshaciael pensamiento (Salmon, 2008). Esto hace que se aumente la capacidad metacognitiva del alumnado, lo que favorece el desarrollo de pensamientos profundosy comprensivos (Perkins, 2016; Ritchhart et al., 2014). Tishman \& Palmer (2005) consideran que las rutinas sirven para realizar una función de diagnóstico al permitir identificar lo que el alumnado sabe de un tema, sus conocimientos previos y su conexión con los nuevos aprendizajes adquiridos (León-Díaz et al ., 2020).

Finalmente hay que señalar que estos patrones de pensamiento contribuyen a la competencia clave aprender a aprender (O rden ECD/65/2015), pues fomentan la autonomía y la autorregulación del al umnado en el proceso de enseñanza-aprendizaje (García, Cañas \& Pinedo, 2017a). Asimismo, en el pensamiento visible, los procesos mentales implicados en el aprendizaje adquieren una gran relevancia. El alumnado debe involucrarse en procesos reflexivos de planificación, de supervisión y de evaluación tanto del resultado como del proceso (Gómez-Barreto, Rubiano-Albornoz \& GilMadrona).

Por otro lado, el currículo de EF de Educación Primaria (Real Decreto 126/ 2014) establece que este área persigue desarrollar la competencia motriz del alumnado, «entendida como la integración de los conocimientos, los procedimientos, las actitudes y los sentimientosvinculadosalaconductamotorafundamentalmente» (p. 48). Esta normativa destaca que no basta con la práctica motriz, sino que se debe potenciar el análisis crítico en las sesiones de EF. Se trata de ser inteligente motrizmente: saber qué hacer, cómo hacerlo, cuándo y con quién. Para ello se deben poner en marcha acciones mentales como percibir, decidir, evaluar, interpretar, etc. , las cuales guardan estrechos vínculos con los movimientos del pensamiento. Así, el alumnado podrá con- trolar las acciones motrices propias y comprender los aspectos perceptivos, cognitivos y emotivos relacionados con la práctica motriz (M oraga, 2011; Xun, 2014; Zhang, 2017).

Cuando exploramos entre la literatura relacionada con la temática en diferentes bases de datos (Dialnet, Scopus y Web of Science), observamos que son reducidos los estudios que se centran en analizar el papel del pensamiento en las sesiones de EF de Educación Prima ria y, de forma específica, las contribuciones del enfoque del pensamiento visible. Por ello, el objetivo concreto que perseguimos con esta investigación es explorar la percepción y la actitud de los profesores de EF vinculados con la etapa de Educación Primaria sobre la promoción y laenseñanza de habilidades del pensamiento del alumnado, así como el conocimiento y la aplicación de estrategias metodológicas para tal fin.

\section{Método}

Este estudio sigue una metodología cualitativa mediante un diseño fenomenológico a través de entrevistas semiestructuradas (Denzin \& Lincoln, 2012).

\section{Participantes}

En el estudio han participado un total de 10 profesores de EF vinculados a la etapa de Educación Primaria. Así, utilizando un muestreo por conveniencia (Flick, 2004; Hernández-Sampieri et al., 2018), inicialmente se solicitó la colaboración a cuatro profesores de la Facultad de Educación de la U niversidad deValladolid de la mención de EF y después a seis profesores que imparten el área de EF en centros educativos públicos de Primaria de Segovia o Soria en el curso 2019/ 2020 o 2020/2021. Las principales características de los participantes se presentan en la tabla 1. 
Tabla 2

Temas de la entrevista semiestructurada

Razones para promover el pensamiento en $E$

Justificación curricular de la promoción del pensamient

0 portunidades del área de EF para promover el pensamiento

Importancia otorgada a la promoción del pensamiento

Herramientas para promover y visibilizar el pensamiento

La promoción del pensamiento en la estructura de la sesión de EF

Enseñanza del pensamiento eficaz: aprender a pensar

Carencias detectadas en el área sobre la temática en cuestión

\section{Procedimiento}

Se realizaron las entrevistas semiestructuradas a aquellos profesores que aceptaron participar en la investigación. La duración media de estas entrevistas fue de 25 minutos. El análisis de los datos cualitativos se llevó a cabo mediante el programa informático Atlas.ti versión 8 siguiendo una categorización emergente (Gibbs, 2012). Para ello, tras transcribir todas las entrevistas, se detectaron las categorías emergentes en un primer nivel de codificación abierta, revisando en todo momento lo analizado cuando emergían nuevos códigos. Después, en un segundo nivel de codificación, se agruparon las categorías en temas para generar teorías, hipótesis y explicaciones sobre las cuestiones de debate. Finalmente, se elaboró un mapa conceptual a partir de los principales resultados.

Todos los procedimientos realizados en este estudio se ajustaron a las normas éticas de los comités de investigación institucionales y/ o nacionales y a la Declara ción de Helsinki y sus posteriores enmiendas o normas éticas comparables (Noreña, Alcaraz-M oreno, Roja \& Rebolledo, 2012).

\section{Resultados}

\section{Razones para promover el pensamiento en EF}

Se destaca que el fenómeno estudiado concuerdacon una concepción integral de la EF que persigue el desa rrollo global de los estudiantes. Los participantes reconocen que las habilidades del pensamiento son necesarias para generar autonomía en el alumnado, consciencia de los aprendizajes y significatividad de estos. «Es fundamental para que los alumnos se conviertan en ciudadanos responsables con capacidad para tomar decisiones propias» (E9). Todas estas razones se corresponden con «las habilidades para la vida demandadas en el siglo $X X \mid »(E 6)$. Asimismo, exponen que otorgar importancia a esta cuestión resulta de interés para aumentar la motivación, el protagonismo del alumnado y tener en cuenta sus intereses. Otros profesores creen que «el pensamiento es inherente al ser humano en todo momento, no concibo no fomentarlo en ningunaárea» (E8).

\section{Justificación curricular dela promoción del pen- samiento}

Todos los entrevistados asocian la promoción y la enseñanza de las habilidades del pensamiento con los procesos cognitivos inherentes a la propia actividad motriz. Así, indican que en los contenidos del área de EF no existe una vinculación explícita a la promoción del pensamiento, puesto que «en la lectura de un contenido de forma implícita van contenidos los tres ámbitos (físico, cognitivo y emocional)» (E5).

Sin embargo, algunos participantes reconocen que el fenómeno de estudio se puede conectar explícitamente con las competencias clave, llegando a mencionar 0 a hacer referencia a la dimensión metacognitiva de la competencia aprender a aprender (E2).

«El alumnado tiene que tener claro qué es lo que está haciendo y cómo. Esto es aprender a aprender» (E4).

«Cuando buscas que analicen sus estrategias» (E9).

«Se trata de que sepan por qué lo hacen, qué lógica tiene hacer esas tareas» (E10).

Gran parte de los entrevistados recurren a la necesidad de no regresar a la tradicional dicotomía entre la cognición y la motricidad en el área de EF. Como reconoce E1: «Desde el momento que se concibe la EF del currículo de manera integrada con todos los elementos de la persona, el pensamiento está siempre presente en todas las sesiones».

\section{Oportunidades del área de EF para promover el pensamiento}

Entre los entrevistados existe pleno consenso sobre las numerosas oportunidades que ofrece el área de EF para promover el pensamiento del alumnado. Incluso, al gunos consideran que este objetivo se puede abordar en mayor medida que en otras áreas. «A mí me parece que es una de las que más puede potenciar el pensa miento si se hace bien porque es una asignatura muy competencial» (E7). También en este sentido, E8 enumera diferentes oportunidades: «las vivencias, la experiencia, las relaciones, los conflictos, los retos de aprendizaje, la conexión consigo mismo y con su cuerpo, con los otros, con el entorno, etc.» Otros consideran que «nos ofrecelasmismas oportunidades que cual quier otra área, siempre y cuando las actividades que hacemos no sean solo una ejecución práctica, sino que tengan un sentido, podamos definir una estrategia, etc.» (E3). En este sentido, E9 hace referencia a la «constante toma de decisiones que hay que tomar a la hora de hacer ciertas 
prácticas de EF, lo cual hace que los alumnos tengan que pensar». E10 expone que: «En EF contamos con la motivación hacia el área y eso hace que los alumnos se sientan relajados y aptos para pensar». A pesar de esta predisposición inicial, «al alumnado hay que motivarle durante la sesión para que pueda profundizar en sus ra zonamientos, sus pensamientos $y$ en su manera de $a c$ tuar posterior» (E4).

\section{Herramientas para promover y visibilizar el pensamiento}

Con respecto a este tema de debate, E10 afirma que «todo gira en torno a la metodología y a la evaluación, los dos pilares básicos de la educación y, en especial, de laEF».

Profundizando en el primero de ellos, los profesores señalan diversidad de herramientas metodológicas para promover las habilidades del pensamiento: Ios modelos pedagógicos, las metodologías activas, los estilos de enseñanza cognoscitivos, las explicaciones iniciales, las paradas de reflexión, las puestas en común o el trabajo através de preguntas. E8 señalaotras, tales como: «Reflexiones, compartir sensaciones, experiencias, retos cooperativos, resolución conjunta de problemas, crear juegos de estrategias... ».

La siguiente cita de uno de los participantes refleja que la metodología condiciona las demandas cognitivas que el alumnado debe involucrar en $\mathrm{EF}$.

«Si empleas en tus clases estilos de enseñanza tradicionales probablemente no se esté fomentando nada el pensamiento. Son estilos de enseñanza que implican una conformidad cognitiva, es decir, el alumno simplemente reproduce lo que se dice. Pero, desde un punto de vista cognitivo, tienes que provocar esa disonancia. Hay otros estilos de enseñanza que rompen esa barrera cognitiva, como la resolución de problemas o el descubrimiento guiado. Ahí es donde el alumno tiene que movilizar todos sus recursos para llegar a los nuevos aprendizajes» (E5).

Entre las herramientas mencionadas, destacan las paradas de reflexión y las asambleas, En estos momentos, E3 considera que «el docente debe plantear buenas preguntas para obligar al alumnado a reflexionar y a decidir». De la misma manera, «debemos saber qué aprendizajes les han quedado claros y cuáles necesitan reforzar» (E2).

«En las reflexiones se debe conseguir que el alumnado tenga un sentido estratégico en las activida des: proponiendo variantes, valorando lo que se está haciendo, valorando cómo se ha realizado, qué elementos son claves, etc.» (E3).

Ahora bien, uno de los participantes manifiesta que: «Para que el pensamiento sea eficaz sobre todo hay una cuestión de partida: tenemos que favorecer la posibilidad de que ese pensamiento se exprese, porque muchas veces algunos no lo expresan» (E3).

Por otro lado, son escasas las alusiones al pensamiento visible, pues predomina su desconocimiento por parte del profesorado de EF o simplemente aplican puntualmente alguna rutina del pensamiento como «veo, pienso, me pregunto» (E7). Como afirma E1: «Q ue el pensamiento visible tal cual como corriente esté en EF ahora es una utopía. Todavía no se ha dado el paso de formar a mæastros en este tipo de metodologías. El pensamiento visible en otros ámbitos es muy reciente». 0 tros exponen que: «He leído algo pero no tengo, no puedo decir que puedo aplicar consecuentemente estas cosas» (E3) 0 «D esconozco si alguna de las rutinas que empleo tienen cabida en este enfoque» (E9).

Sobre la evaluación, son varias las alusiones a las técnicas de evaluación formativa, tales como: la autoevaluación y la coevaluación. «Hay que introducirlas para fomentar el pensamiento individual, pero también la observación entre iguales con técnicas adecua das, lo que favorece que reflexionen sobre lo que hacen y pueden hacer» (E10).

Por otro lado, uno de los participantes sugiere que, a modo de tarea, se puede demandar al alumnado la realización de una actividad de evaluación que tenga como objetivo el análisis crítico de lo realizado de forma práctica. «Cabe la opción de que dentro de los instrumentos de evaluación que planteamos como área haya una parte de reflexión para que lo puramente práctico no termine en la ejecución ni en la propia sesión, sino que tenga un componente de análisis y de reflexión que complementaría todo lo anterior» (E3).

\section{La promoción del pensamiento en la estructura de la sesión de EF}

Como ya se ha comentado, los participantes desta can el papel de las asambleas y de las paradas de reflexión durante la estructura de la sesión. De hecho, la mayoría propone que la estructura de la sesión más idónea para promover el pensamiento es la que consta de tres momentos: la asamblea inicial, la actividad motriz y la asamblea final.

Sobre la relevancia real que se otorga a cada uno de tres momentos mencionados, E10 señala que, a veces, únicamente se otorga importancia a la segunda fase: la 
actividad motriz. Según él, «aunque para muchos compañeros no siempre es así, para mí tanto la actividad motriz como la reflexión tienen la misma relevancia» (E10).

En este sentido, algunos profesores señalan que estos momentos de reflexión no se suelen planificar lo suficiente. E3 defiende la necesidad de que en estas situaciones de reflexión se tenga una «previsión hacia dónde orientar las paradas, las preguntas preparadas, etc.». Es decir, se refiere al diseño previo a la sesión con el alumnado de las preguntas inteligentes que se les realizarán para hacerles pensar. «La reflexión no se rea liza sin más, sino planificando y pensando cómo vamos a desarrollar estas actividades» (E10).

\section{Enseñanza del pensamiento eficaz: aprender a pensar}

Al preguntar a los participantes sobre las posibles diferencias entre promover el pensamiento y enseñar a pensar de forma eficaz, sus respuestas han sido bastante diversas.

«Pues creo que enseñarles a pensar va mucho más dirigido, o sea, enseñamos al alumnado a llegar a un objetivo. Promover el pensamiento es que ellos mismos desarrollan su consciencia crítica y no les guíes tanto» (E7).

«Considero que promover el pensamiento es hacer que los alumnos sean capaces de cuestionarse las cosas por ellos mismos, mientras que pensar de forma eficaz es orientar a los al umnos para que sus pensamientos se dirijan hacia el punto que ellos quieren» (E9).

«Bueno, puede que sí haya diferencias. U na cosa es promover, incluso puedesguiar su pensamiento... y otra bien distinta es desarrollar autonomía para que durante la clase de EF se generen pensamientos, razonamientos y situaciones de cambio, donde se constate que los alumnos maduran en el juego, que entienden su estructura, su rol y su actividad» (E10).

«Yo creo que es bastante parecido. El trabajar el pensamiento y el reforzar sus creencias y sus acciones sobre la reflexión sobre lo realizado» (E3).

En relación con estas ideas que se discutirán posteriormente, E3 destaca que «un aspecto clave es el feedback que los docentes damos al alumnado, pues les podemos orientar para que no se queden en lo superficial y desarrollen un pensamiento eficaz» (E3).

\section{Carencias detectadas en el área sobre la temá- tica en cuestión}

Los participantes señalan que la promoción del pen- samiento del alumnado no es frecuente en EF, pero tampoco en el resto de las áreas curriculares. No obstante, como reconocen varios de los profesores, «todo depende del centro educativo y, sobre todo, de los propios docentes y de su profesionalidad» (E3). E9 también expresa:

Creo que como regla general no se promueve el pensamiento en casi ninguna asignatura. En EF por lo que puedo apreciar tampoco se promocionamucho, todo depende de la metodología empleada por el docente (E9).

Con respecto a la EF, E3 expone que: «En los últimos años ha habido un salto cualitativo para dotar de rigor a la materia que antes no estaba tan fundamenta do. Sin embargo, este cambio no se acompaña tanto en la práctica en los centros educativos». Ante esta idea, los entrevistados exponen una doble perspectiva sobre la orientación de la EF. Por un lado, aquella que se centra en la aplicación de «estilos de enseñanza tradiciona les, en la repetición de ejecuciones motrices y en la realización de meros juegos motrices» (E2). Esta perspectiva reduce la EF a lo motriz debido «a la herencia negativa del área y a la escasa importancia que se le ha otorgado en el sistema educativo» (E5).

La reducción de la EF a lo motriz deja de lado otras cuestiones como la promoción del pensamiento de los estudiantes. Esta idea que queda recogida por varios de los profesores:

«Considero que en EF tradicionalmente se ha dado más importancia a otros aspectos (lúdicos, actitudinales o motrices) dejando a un lado otros aspectos de índole más cognitiva, por lo que los temas relacionados con el pensamiento y la reflexión todavía están muy infravalorados» (E9).

«El pensamiento tiene que ver con que todas las acciones tengan una valoración. No limitarnos a realizar acciones 0 a actuar por actuar, sino dar un sentido y analizar en profundidad lo que hacemos y para qué lo hacemos. De esta manera, el alumnado será consciente del proceso formativo de la asignatura» (E3).

«A veces es convertir las clases en una sucesión de actividades... A ver cómo completo la hora para que estén entretenidos y trabajen el contenido que yo me he marcado, pero realmente no hay esa fuerza reflexiva que hace pues profundizar más en lo que se está haciendo tanto en el profesor como en el alumno» (E4).

«En los centros mi experiencia es que no se promueve el pensamiento porque hay unas rutinas y unos hábitos que se están desarrollando desde hace décadas. Es una reproducción del sistema, de lo que se ha hecho 
tradicionalmente. No se pone en valor lo que realmente interesa: saber cuánto y qué aprenden los alumnos» (E6).

En segundo lugar, aquella orientación de la EF propia del siglo XXI con finalidades claras de aprendizaje, a la vez que formativa. Desde esta perspectiva, se favorece la promoción del pensamiento del alumnado y contribuye a mejorar el estatus de la asignatura. E1 considera que, si no se hace explícita la vinculación entre EF y pensamiento, «los niños, y muchos profesores, se van creyendo que lo motriz es exclusivamente motriz. Todo esto teniendo cuidado de no irnos solo a lo cognitivo y olvidarnos de lo motriz» (E1).

Ante esta situación, los entrevistados también reconocen ciertas carencias en el profesorado en lo que respecta al conocimiento de herramientas para dar respuesta al fenómeno estudiado, señalando la necesidad de que se conozcan en la formación inicial y permanente de los docentes. Como afirma E6, «solo la formación personal hace que podamos mejorar nuestros conocimientos». N o obstante, E3 tiene unavisión opuesta, pues considera que no es un problema de formación, sino de tradición, actitud y comodidad: «Todos los docentes, en mayor o menor medida, tienen capacidad para sumarse a conseguir una EF más formativa utilizando dinámicas en las cuales se favorezca la formación consciente y reflexiva del alumnado. O tra cuestión es que por comodidad se promueva solo valorar el componente físico y seguir otro modelo» (E3).
En este sentido, los participantes consideran que el fenómeno estudiado requiere de predisposición por parte del docente para otorgar importancia al pensamiento en el aula. El docente «debe actuar como guía» (E1), «haciendo conscientes a los estudiantes de la importancia de las habilidades del pensamiento» (E6). Sobre esta situación, E4 resalta a veces no se promueve el pensamiento del alumnado por la inseguridad que se pueda generar en el profesorado ante las preguntas o los intereses del alumnado. «Cuando damos opción a que el alumno reflexione puede decirnos cosas que no tenemos preparadas y hay que responder... Cuando te ponen en un frente que no sabes por dónde salir, esta inseguridad o miedo conduce a no fomentar el pensamiento del alumnado» (E4).

También algunos de los entrevistados destacan que el fenómeno de estudio puede no estar del todo presente debido al reducido «tiempo útil que dispone el área de EF en cada una de las sesiones» (E6). «N o se suele introducir en las aulas por el poco tiempo del que cada vez disponemos, anteponiendo el movimiento tal cual sin reflexión» (E10).

Los principales resultados cualitativos se recogen en la figura 1.

\section{Discusión}

Los datos de las entrevistas cual itativas reflejan que los participantes consideran relevante la promoción y

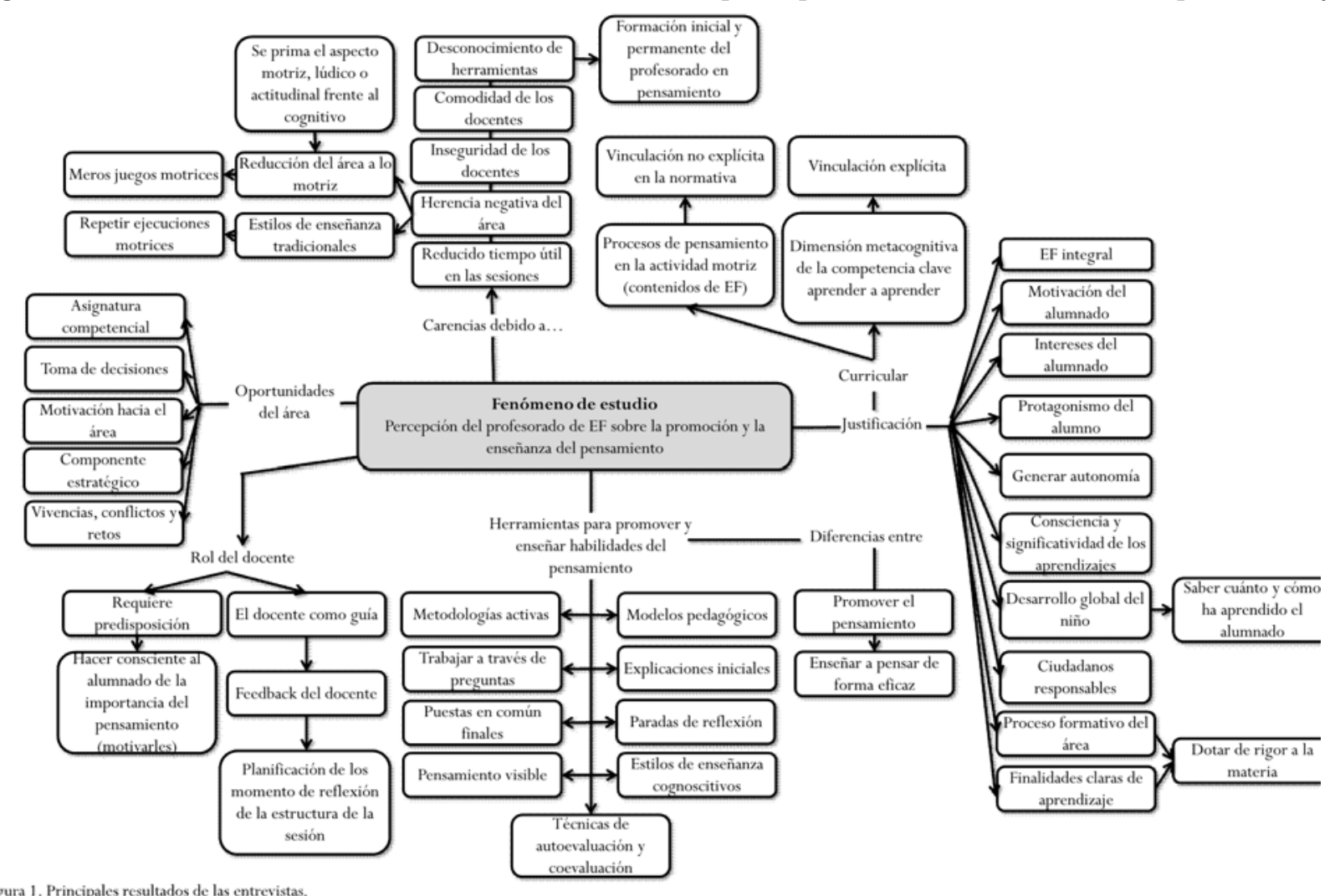


la enseñanza de las habilidades del pensamiento en las sesiones de EF, señalando para ello diversas razones a nivel pedagógico o curricular. No obstante, la mayoría de sus respuestas justifican este fenómeno haciendo referencia a los procesos cognitivos inherentes a la actividad motriz 0 a los contenidos curriculares de EF. Con este estudio no se pretende regresar a la tradicional dicotomíaentre motricidad y cognición, sino que se parte de una concepción integral de la EF sobre la cual desa rrollar el pensamiento profundo del alumnado (Ritchhart et al., 2014) y la dimensión cognitiva de la competencia motriz (Giménez, López-Téllez \& Sierra, 2009; Real Decreto 126/ 2014).

Las herramientas metodológicas que señalan los participantes concuerdan con las contribuciones de los autores que defienden el uso de estrategias que promuevan la participación activa y reflexiva del alumnado y su implicación en la toma de decisiones en EF (León-Díaz et al. , 2020, Pill \& Suee, 2017; Zueck, Alonso, Ramírez, Margarita \& Irigoyen, 2020).

Sin embargo, la mayoría de las herramientas que los participantes señalan encajan con la perspectiva de la enseñanza del pensamiento basada en la inmersión (Jonhson, 2003). Esto se debe a que estas herramientas enfrentan al alumnado a situaciones que invitan a pensar, estableciéndose una atmósfera de reflexión a partir de la cual se espera que los estudiantes desarrollen ha bilidades del pensamiento profundo mediante los contenidos curriculares (Perkins, 2016). Es decir, ofrecen situaciones u oportunidades que fomentan que el al umnado piense, lo cual no significa que vaya a ocurrir y tampoco que vayan a producirse procesos cognitivos eficaces (M orales \& Restrepo, 2015). Algunas de estas herramientas son: los retos cooperativos, el estilo actitudinal, la elaboración de estrategias en los juegos, la resolución de problemas, el compartir sensaciones, etc.

Son escasas las al usiones al pensamiento visible 0 al uso de las rutinas del pensamiento por parte de los participantes, las cuales son herramientas metodológicas que se engloban dentro de la enseñanza infusionada del pensamiento (Swartz et al., 2017). Desde esta perspectiva se aboga no solo por promover el pensamiento, sino también por la enseñanza de las habilidades del pensamiento para que el alumnado desarrolle un pensa miento eficaz gracias al repertorio de estrategias cognitivas trabajadas (Baez \& 0 nrubia, 2016). Paraello, es crucial permitir que los pensamientos se expresen 0 visibilicen (Salmon, 2015).

Asimismo, mediante un proceso de andamiaje por parte del profesor, se deben fomentar todos los movimientos del pensamiento y estructurar las reflexiones de las asambleas, las paradas de reflexión u otras tareas en torno a ellos (Hamada, 2015). En este sentido, como han reflejado algunos participantes, se requiere de la planificación previa de estos momentos por parte del profesor (Pill, 2016). El al umnado debe tener consciencia de los movimientos del pensamiento que desempeñan. Cuanto más explícita sea la enseñanza del pensa miento, mayor seráel impacto en los estudiantes (M aina, Schlegel y Hunt, 2016; Lodewyk, 2009; Swart et al., 2017). En la figura 2 se resumen estas ideas expuestas.

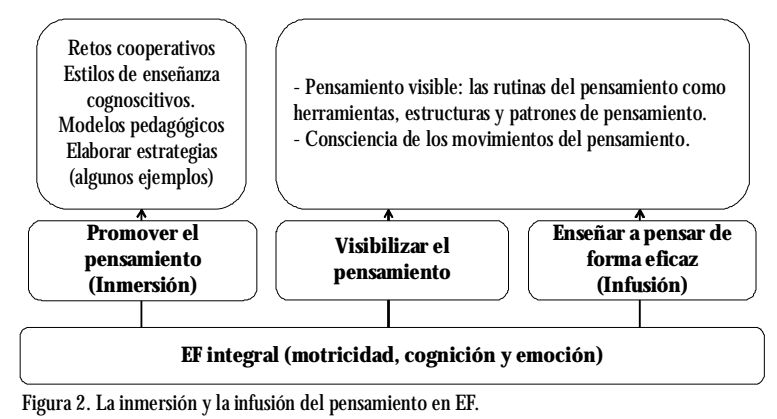

En cualquier caso, tanto las herramientas metodológicas propias de una enseñanzadel pensamiento basada en la inmersión como las propias de la enseñanza infusionada son compatibles. Ambas perspectivas permiten al al umnado generar disposiciones positivas hacia el hábito de pensar (Swartz et al., 2017). Esto se debe a que pensar no es un acto mecánico, requiere de esfuerzo por parte del que piensa (Perkins, 2016).

Aunque de forma intencionada o intuitiva el profesorado de EF persiga la promoción del pensamiento, el nivel de consecución de este objetivo aumentaría si se contase con conocimientos sobre el enfoque del pensa miento visible: las fuerzas culturales, las rutinas del pensamiento y losmovimientos del pensamiento (Pill, 2016; Ritchhart et al., 2014; Ritchhart, 2015; Salmon, 2015; Sanz, 2018). La EF se mejora cuando se enseña con una consciencia de los resultados hacia los que se está enseñando (Liu et al., 2018).

Por tanto, el objetivo es triple: promover el pensa miento (situaciones que favorezcan el pensamiento de los estudiantes), visibilizar las reflexiones o las ideas del alumnado (permitir que se expresen) y enseñarles a pensar (dotarles de habilidades metacognitivas) (RuizMartin, 2020).

Los programas de formación del profesorado deberían hacer conscientes a los docentes de que no basta con enfrentar al alumnado a situaciones que demanden pensar, sino que es preciso enseñarles a pensar de forma 
eficaz. Para ello, es fundamental a aumentar la confianza del profesorado con respecto al trabajo del pensa miento. El profesor desempeña un papel fundamental en la motivación, las estrategias de aprendizaje y el desarrollo del pensamiento crítico del al umnado (Trigueros \& Navarro, 2019).

Por otro lado, los docentes que han participado en las entrevistas reconocen carencias en el área de EF en lo que respecta a la promoción y la enseñanza de habilidades del pensamiento del alumnado. Es decir, a pesar de que lo consideran importante y reconocen las posibilidades formativas del área para tal fin, detectan que a veces se otorga mayor o exclusiva importancia al movimiento y a la actividad motriz.

Este hecho puede deberse a diversas razones: la falta de conocimiento sobre herramientas metodológicas activas que fomentan y enseñan habilidades del pensamiento (León-Díaz et al., 2020); las dificultades o la inseguridad del profesorado en la interacción con el alumnado, en el uso y la gestión del feedback correctivo y de carácter formativo (Asún, Fraile, Aparicio y Romero, 2020); el reducido tiempo útil de las sesiones de EF (Ruiz, Lara, López, Cachón \& Val divia, 2019); la herencia negativa del área de $\mathrm{EF}$, manifestándose a través del predominio del mero entrenamiento físico, la falta de programación o de secuencias claras, etc. (López, 2005); y, en relación con la idea anterior, la tendencia a reproducir el modelo vivido por parte del profesorado durante su etapa como estudiante (López, Pérez, Manrique \& M onjas, 2016).

En cualquier caso, como se comprobaráseguidamente, el pensamiento visible puede conver tirseen un marco global en el cual englobar otras herramientas existentes en el área de EF. Este enfoque reforzaría las finalida des de aprendizaje de EF en el siglo XXI (López et al., 2016), contribuyendo a la innovación educativa, es decir, aquella que impacta generando aprendizajes de calidad en el alumnado (Pérez-Pueyo \& Hortigüela, 2020).

\section{Consejos para introducir el pensamiento visible en Educación Física}

Para instaurar una cultura del pensamiento se deben contemplar las ocho fuerzas culturales propuestas por Ritchhart (2015).

1. Altas expectativas hacia el pensamiento. Esta fuerza cultural se vincula con la dimensión actitudinal. Los estudiantes deben tener claro que en las clases de EF se valora su pensamiento para al canzar la comprensión sobre aquello que están realizando.
2. Oportunidades para promover el pensamiento. Como se ha mencionado, se debe planificar el trabajo explícito de los movimientos del pensamiento en la programación docente, de tal forma que todos ellos estén presentes a lo largo de un proyecto 0 de una unidad didáctica. Para ello, se puede utilizar la lista de cotejo que proponen- Pinedo, Cañas \& García (2017b). Por otro lado, las situaciones 0 las actividades de aprendizaje diseñadas tienen que favorecer que el alumnado tenga que pensar. Para ello, se pueden utilizar diversas herramientas metodológicas propias de la perspectiva de la inmersión del pensamiento: resolución de problemas, descubrimiento guiado, aprendizaje cooperativo, estilo actitudinal, etc. Los modelos pedagógicos son herramientas del proceso de al fabetización física. U na de las características de dicha praxis alfabetizadora es el pensamiento crítico y la incidencia en el ámbito cognitivo (Fernández, Méndez \& Sánchez, 2018).

3. Rutinas del pensamiento. Estas estructuras de pensa miento se pueden utilizar durante los momentos de reflexión de la estructura de la sesión: en la asamblea inicial, en las paradas de reflexión-acción y en la asamblea final. Se pueden proponer al alumnado a partir de las situaciones cognitivas generadas con la fuerza cultural oportunidades. En la tabla 3 proporcionamos algunos ejemplos en función del objetivo de cada una.

Como se comprueba, las rutinas del pensamiento proporcionan un lenguaje eficaz al alumnado, así como estrategias cognitivas que los estudiantes pueden interiorizar y transferir posteriormente a otras situa-

Tabla 3.

Algunos ejemplos de rutinas del pensamiento

Objetivo dela

bjetivo de

rutina del

pensamiento

Rutinas para Tras presentar inicialmente un determinado contenido, los estudiantes

presentar y deben pensar tres ideas, dos preguntas y una metáfora o analogía sobre él. Se

explorar ideas repite esta actividad tras realizar una actividad sobre el contenido en cuestión trabajado en el aula.

Rutinas para Antespensabo hhor pienso

sintetizar y Tras abordar en el aula un deter minado contenido, el alumnado debe

organizar ideas plantear se cómo ha evolucionado su pensamiento.

Afirma, a poya, cuestiona

Rutinas para Sobre un determinado contenido, el alumnado elabora una afirmación. explorar las ideas Posteriormente, los estudiantes deben identificar evidencias que la de forma profunda justifiquen $y$, finalmente, se deben plantear una pregunta que esté relacionada con su afirmación.

\section{Hablar-Preguntar-Ideas-Aprendizajes}

Un estudiante comparte su idea sobre un determinado plan de acción ante un reto o problema. El grupo debe realizar preguntas de aclaración al

Rutinas para un reto o problema. El grupo debe realizar preguntas de aclaración al
estudiante. Después, el grupo debe ofrecer ideas para mejorar la idea inicial

relacionarse con estudiante. Después, el grupo debe ofrecer ideas para mejorar la idea inicial

los demás en este momento ninguna valoración de las propuestas. Cuando todos los compañeros finalizan, el estudiante expresa aquello que ha aprendido, señalando cualquier pensamiento nuevo sobre el plan. Nombrar-Describir-Actuar

Rutinas para Desde esta rutina del pensamiento se persigue que el alumnado haga una relacionar se con las lista de todas las partes 0 características sobre un determinado contenido

ideas trabajado. Tras ello, los estudiantes deben describir cada una de las partes mencionadas y, finalmente, explicar cuál es la función de cada uno de ellos.

Predecir-Reunir-Explicar

Rutinas para Sobre una situación o problema, el alumnado debe predecir cuáles serán los

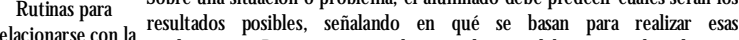

acción la predicciones. Posteriormente, los estudiantes deben recopilar datos información sobre el problema. Finalmente, tienen que dar sentido a los datos recopilados e interpretarlos en relación con las predicciones iniciales. Fuente: elaboración propia a partir de Ritchhart et al. (2014) o en Ritchhart y Church (2020). 
ciones (Salmon, 2008). Para adquirir un conocimiento más profundo sobre estos patrones del pensamiento se pueden consultar las publicaciones de Ritchhart et al. (2014) o de Ritchhart y Church (2020).

A lo largo de un proyecto de aprendizaje o unidad didáctica se deben introducir progresivamente diversos patrones de pensamiento. El alumnado debe saber qué se pretende conseguir con cada una de las rutinas del pensamiento antes de que las realicen: cuál es el objetivo y qué movimientos del pensamiento desempeñan.

Como estos patrones de pensamiento se pueden tra bajar de forma oral y escrita, durante la sesión se puede otorgar importancia a realizar las rutinas del pensamiento forma oral para aprovechar el tiempo de implicación motriz. Tras la sesión, se puede demandar al alumnado la realización de una rutina del pensamiento como ta rea específica en un cuaderno o portafolio de la asignatura. Además de otro cauce de participación para el al umnado, Llamas (2008) demuestra que el cuaderno 0 portafolio es un instrumento de evaluación formativa que permite desarrollar la reflexión por parte del alumnado.

4. Modelaje. El docente debe ser un modelo para el alumnado en cuanto al uso de los movimientos del pensamiento durante las sesiones. Esto significa que el profesor debe participar en las rutinas del pensamiento que se plantean en el aula, mostrar curiosidad por las preguntas que el alumnado se realiza, dar importancia a las reflexiones, etc.

5. Lenguaje. Dado que el lenguaje modela el pensa miento, durante las sesiones se debe emplear un lenguaje propio del pensamiento eficaz: (a) verbos que fa ciliten el pensamiento (e.g. «elaborar», «evaluar», «justifica», «explicar», «comparar y contrastar», etc.), (b) frases condicionales (e.g. «podría ser», «debería ser», «una posibilidad es», etc.); (c) preguntas (e.g. ¿Q ué prevéis que sucederá... ? ¿Qué conclusiones... ? ¿Cuál es el objetivo de... ? ¿Q ué pruebas apoyan... ? ¿Q ué podéis deducir... ?, etc.), y (d) hacer notar y nombrar el pensamiento que ocurre en el aula haciendo alusión a los movimientos del pensamiento (e.g. «Manuel está estableciendo conexiones con sus experiencias previas para apoyar su razonamiento» 0 《R uth está contemplando diferentes alternativas»).

6. Tiempo para pensar. Aunque sea de forma breve, se debe dedicar tiempo a explicar los movimientos del pensamiento que se trabajan con las rutinas del pensa miento, así como a poner nombre a los procesos cognitivos en los cuales se involucra el alumnado. Esto permite desarrollar su capacidad metacognitiva. Por otro lado, el buen pensamiento requiere tiempo, por lo que se debe otorgar tiempo al alumnado para que piense. En los momentos de reflexión el profesor no puede dominar la conversación, losal umnos deben ser los protagonistas a través de sus pensamientos, sus ideas y sus reflexiones.

7. Ambiente físico. Se puede diseñar un rincón o mural del pensamiento de EF en el cual registrar todas las rutinas del pensamiento que se demandan al al umnado. No debe ser una mera exposición estática de estos tra bajos, sino que se puede aprovechar para explicar determinados movimientos del pensamiento en las para das de reflexión o en los momentos de asamblea. Se trata de un rincón que inspira a pensar al alumnado.

8. Interacciones entre el al umnado. Las interacciones no son únicamente beneficiosas para generar un clima cooperativo o colaborativo en el aula, sino que son necesarias desde un punto de vista del pensamiento. Se pueden aplicar técnicas cooperativas (Velázquez, 2010), plantear rutinas del pensamiento para apoyar la toma de decisiones grupales o la indagación, fomentar la escucha entre compañeros, etc.

\section{Conclusiones}

Este estudio demuestra que, aunque el profesorado considera necesario el desarrollo del pensamiento profundo del alumnado, la herencia negativa del área, la falta de formación docente o la inseguridad del profesorado hacen que en las sesiones de EF se otorgue mayor importancia a la actividad motriz frente a la promoción y la enseñanza de las habilidades del pensamiento.

El pensamiento visible puede constituirse como un marco global desde el cual promover el pensamiento del alumnado, a la vez que se les enseña a pensar de forma eficaz. De esta forma, el uso regular de las rutinas del pensamiento podría permitir que el alumnado interiorizase los movimientos del pensamiento como patrones de comportamiento.

Innovar en EF mediante la integración del enfoque del pensamiento visible contribuiría a reforzar las finalidades de aprendizaje del área de EF del siglo XXI, la competencia clave aprender a aprender y el desarrollo de la competencia motriz del alumnado.

\section{Referencias}

Assessment \& Teaching of 21st Century Skills. (2014). Competencias del sigloXXI. Guía práctica para promover su apren- 
dizaje y evaluación. Springer.

Asún, S. , Fraile, A.,Aparicio, J. L., \& Romero, M. R. (2020). Dificultades en el uso del feedback en la formación del profesorado de Educación Física. Retos. Nuevas tendencias en Educación Físca, Deportey Recreación, 37, 85-92.

Báez, J., \& O nrubia, J. (2016). U narevisión de tresmodelos para enseñar las habilidades de pensamiento en el marco escolar. Perspectiva Educacional, Formación de Profesores, 55(1), 94-113. Recuperado de https: / / bit. ly/ 3864eAg

Bamberger, M. (2012). Introducción a los métodos mixtos de la evaluación de impacto. N otas sobre la Evaluación de Impacto, 3, 1-42. Recuperado dehttps:/ / bit.ly/ 2X07tTB

Cabañete, D., Tesouro, M., Puigggali, J., \& Zagalaz, M. L. (2019). Estado actual delaEducación Físicadesdeel punto de vista del profesorado. Propuestas de mejora Retos Nuevas tendencias en Educación Físca, Deporte y Recreación, 35, 47-53.

Dede, C. (2010). Comparing frameworks for 21st century skills. 21st century skills: Rethinking how students learn, 20(2010), 51-76. Recuperado de https:/ / bit. ly/ $38245 \mathrm{hw}$

Denzin, N. K., \& Lincoln, Y. S. (2012). Manual de investigación cualitativa. Barcelona: Gedisa.

Fernández, J., Méndez, A., \& Sánchez, R. (2018). Didáctica de la educación física para bachillerato basada en modelos. Madrid: Síntesis.

Flick, U. (2004). El diseño delnvestigación Cualitativa. Madrid: Morata.

García, N., Cañas, M., \& Pinedo, R. (2017). Innovación educativa y Pensamiento Visible en Educación Primaria. En M. Pinos(Ed.), Libro deactas del I Congreso Internacional deInnovación Educativa, Zaragoza(España) (pp. 1-13). Za ragoza, España: Gobierno deAragón.

Gibbs, G. (2012). El análisisdedatos en investigación cualitativa. Madrid: M orata.

Giménez, A. M., López-Téllez, G., \& Sierra, B. (2009). Competencias básicas: sobre la exclusión de la competencia motriz y las aportaciones desde la Educación Física. Retos Nuevastendenciasen Educación Física, D eportey Recreación, 16, 51-57.

Gómez-Barreto, I., Rubiano-Albornoz, E. \& \& Gil-Madrona, P. (2019). Manual para el desarrollo dela metodología activa y el pensamiento visble en el aula. Madrid: Pirámide.

Hamada, M. (2015). Game-based learning and usingVisible Thinking Routines - asking meaningful questions. Active + Healthy Journal, 22(2), 7-12.

Hernández, R., Fernández, C., \& Baptista, P. (2014). M etodología dela investigación. M éxico: M c Graw Hill Education.

Johnson, A. (2003). El desarrollo delashabilidades de pensamiento. Aplicación y planificación para cada disciplina. BuenosAi- res: Troquel.

León-Díaz, 0., Arija-M ediavilla, A., Fernando-Martínez, L., \& Santos-Pastor, M. L. (2020). Las metodologías activas en Educación Física. Una aproximación al estado actual desde la percepción de los docentes en la Comunidad deM adrid, Retos N uevastendenciasen Educación Física, Deportey Recreación, 38, 587-594.

Liu, J., McBride, R., Xiang, P., \& Scarmardo, M. (2018). Physical Education Pre-serviceTeachers' U nderstanding, Application, and Development of CriticalThinking Ques, 70(1), 12-27. Recuperado de https: / bit. ly/ 2X31y00

Llamas, J. (2008). Cuaderno o dossier del alumno. Tándem. Didáctica dela Educación Física, (26), 121-122.

Lodewyk, K. (2009). Fostering Critical Thinking in Physical Education Students. Journal of Physical Education, Recreation \& Dance, 80(8), 12-18. Recuperado de https:/ / bit.ly/ 3rKjqLI

López, V. L. (2005). ¿Q ué Educación Física hemos vivido? Analizando nuestro saber profesional a partir de historias de vida. Lúdica Pedagógica, 2(10), 13-18. Recupera do https:/ / bit.ly/ 34ZitVV

López, V. M., Pérez, D., Manrique, J. C., \& Monjas, R. (2016). Los retos de laEducación Física en el siglo XXI. Retos: nuevas tendencias en Educación Física, deportey recreación, 29, 182-187.

Maina, M. ., Schlegel, M., \& Hunt, K. (2016). InitiativeGames in Physical Education: A Practical Approach for Teaching Critica Thinking Skills - Part II. Strategies 29(4), 8-14. Recuperado de https:/ / bit.ly/ 34X Ivan

M oraga, J. (2011). Enseñanza del pensamiento aplicada a la educación física y a los deportes. Revisa M otricidad y Persona: serie de estudios, 9, 41-45. Recuperado de https:/ / bit.ly/ 3n5kKF8

M orales, M., \& Restrepo, I. (2015). Hacer visible el pensa miento: alternativa para una evaluación para el aprendizaje. Infancias imágenes, 14(2), 89-100. Recuperado de https: / / bit.ly/ 380WorQ

Noreña, A. L., Alcaraz-M oreno, N., Rojas, J. G., \& Rebolledo, D. (2012). Applicability of the Criteria of Rigor and Ethicsin Q ualitative Research. Aquichan, 12(3), 263-274.

OECD. (2001). Competencies for the Knowledge Economy. Recuperado de https:/ / bit.ly/ 3n2mjnb

O pazo, H. (2011). Éticaen investigación: desdelos códigos deconductahacialaformación del sentido ético. REICE. Revista Iberoamericana sobre Calidad, Eficacia y Cambio en Educación, 9(2), 61-78. Recuperado https:/ / bit.ly/ 2LbYAUW

O rden ECD/ 65/ 2015, 5, de 21 de enero, por la que se describen las relaciones entre las competencias, los con- 
tenidos y los criterios de evaluación de la educación primaria, la educación secundaria obligatoria y el bachillerato (BOE núm.25, de 29 de enero de 2015).

Pérez-Pueyo, A., \& Hortigüela, D. (2020). ¿Y si toda la innovación no es positiva en Educación Física? Reflexiones y consideraciones prácticas. Retos, nuevastendencias en Educación Física, deportey recreación, 37, 639-647.

Perkins, D. (2016). Educar para un mundo cambiante. ¿Quénece sitan aprender realmente los alumnos para el futuro? Barcelona, España: SM.

Pill, S., \& SueSee, S. (2017). Including Ceritica Thinking and Problem Solving in Physical Education. Journal of Physical Education, Recreation and Dance, 88(9), 43-49. Recuperado de https: / bit.ly/ 3pLBFyp

Pinedo, R., Cañas, M., \& García, N. (2017a). The development of a checklist for getting «good materials to promote deep thinking in classs. En L. Gómez, A. López y I. Candel (Eds.), Proceedings of ICERI2017 Conference (pp. 4532-4536). Sevilla, España: IATED.

Pinedo, R., Cañas, M., García, N., \& García, N. (2019). Capacidad metacognitiva en docentes y futuros docentes de enseñanza no universitaria. Revista de Psicología y Educación, 14(1), 74

Real Decreto 126/ 2014, de 28 de febrero, por el que se establece el currículo básico de la Educación Primaria (BOE núm.52, de 1 de marzo de 2014).

Ritchhart, R. (2015). Creating cultures of Thinking.The 8 Forces We Must Master to Truly Transform Out Schools. Jossey-Bass.

Ritchhart, R., \& Church, M. (2020). The power of making thinking visible. Practices to engage and empower all learners. San Francisco: Jossey-Bass.

Ritchhart, R., \& Perkins, D. (2008). M kingThinkingVisible. Educational Leadership, 65(5), 57-61. Recuperado de https:/ / bit.ly/ 38523NK

Ritchhart, R., Church, M., \& Morrison, K. (2014). Hacer visible el pensamiento. Cómo promover el compromiso, la comprensión y la autonomía de los estudiantes BuenosAires, Argentina: Paidós.

Ruiz, C. M., Lara, A. J., López, F. J., Cachón, J., \&Valdivia, P. (2019). Análisis del tiempo de clase en EF y propuestasparasu optimización. Retos. N uevastendencias en Educación Física, Deportey Recreación, 35, 126-129.

Ruíz-M artín, H. (2020). ¿Cómo aprendemos? U na aproximación científica al aprendizajey la enseñanza. Barcelona: Graó.

Salmon, A. (2008). Promoting a Culture of Thinking in the Young Child. Early Childhood Education Journal, 35, 457461. Recuperado de https:/ / bit. ly/ 382810s

Salmon, A. (2015). El desarrollo del pensamiento en el niño para escuchar, hablar, leer y escribir. Leer Escribir y D escubrir,2(1), 2-12. Recuperado dehttps: / / bit.ly/ 3aVZwqQ
Sanz, C. (2018). La enseñanza basada en el pensamiento en Educación Infantil: un estudio de casos en la provincia de Segovia (Trabajo Fin de Máster). Universidad deValladolid, Fa cultad de Educación de Segovia.

Scott, C. L. (2015). El futuro del aprendizaje, ¿Quétipo de pedagogías se necesitan para el siglo XXI? Investigación y Prospectiva en Educación UNESCO. París. Recupera do de https:/ / bit.ly/ 2Mh9JUo

Swartz, R., Costa, A., Beyer, B., Reagan, R., \& Kallick, B. (2017). El aprendizajebasado en el pensamiento. Cómo desarroIlar en los alumnos las competencias del siglo XXI. Barcelona, España: SM.

Tishman, S. \& Palmer, P. (2005). Pensamiento visible. Leadership compass, 2(4), 1-3. Recuperado de https:/ / bit. ly/ 2LfGr83

Trigueros, R., \& Navarro, N. (2019). La influencia del docente sobre la motivación, las estrategias de aprendiza je, pensamiento crítico y rendimiento académico de los estudiantes de secundaria en el áreade Educación Física. Psychology, Society and Education, 11(1), 137-150. Recuperado de https:/ / bit.ly/ 2X0]3cH

Voogt, J., \& Pareja, N. (2012). A comparative analysis of international frameworksfor 21st century competences: Implications for national curriculum policies, Journal of CurriculumStudies, 44(3), 299-321. Recuperado dehttps:/ / bit.ly/ 307eoX0

Xun, Z. (2014).TheExploration of the Relationship between Students'Thinking Ability and Sports Skill Formation in the Physical Education. Web of Conferences 7, 14-18. Re cuperado de https:/ / bit.ly/ 3hyokGu

Zhang, X. (2017). On the application of thinking guidance in College Physical Education and training. Adavances in Economics, Business and Management Research, 29(1), 109114.

Zueck, M., Alonso, A., Margarita, J., \& Irigoyen, G. (2020). Satisfacción en las clases de Educación Física y la intencionalidad de ser activo en niños del nivel de primaria, Retos. Nuevastendencias en Educación Físca, Deporte y Recreación, 37, 33-40.

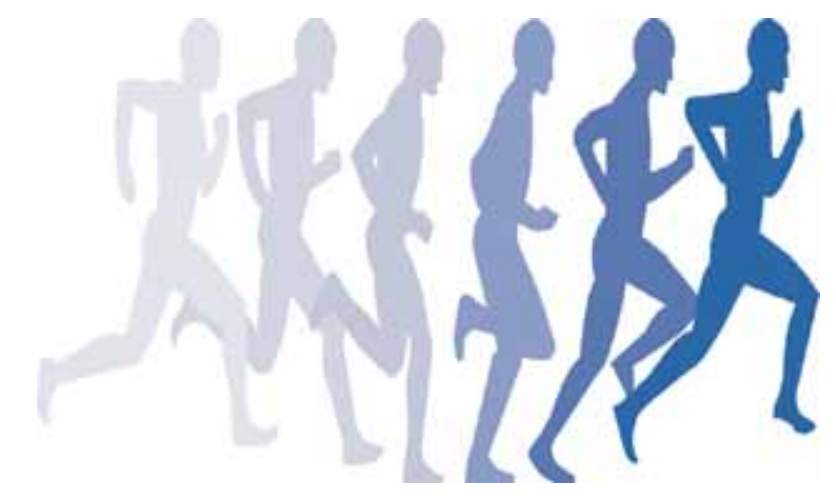

